\title{
Como os profissionais de educação física se reinventaram durante a pandemia do COVID-19
}

\author{
How physical education professionals reinvented themselves during the COVID-19 pandemic \\ Cómo se reinventaron los profesionales de la educación física durante la pandemia del COVID-19
}

Recebido: 24/09/2021 | Revisado: 30/09/2021 | Aceito: 30/09/2021 | Publicado: 03/10/2021

\author{
Angélica Ferreira de Araújo \\ ORCID: https://orcid.org/0000-0001-6678-8484 \\ Centro Universitário Natalense, Brasil \\ E-mail: angelica.ferreira272@hotmail.com \\ Josikleiton Dias de Lima \\ ORCID: https://orcid.org/0000-0003-2321-4397 \\ Centro Universitário Natalense, Brasil \\ E-mail: josikleiton@yahoo.com.br \\ Theógenes Tértius Lima \\ ORCID: https://orcid.org/0000-0001-7326-4443 \\ Universidade Federal do Rio Grande do Norte, Brasil \\ E-mail theogenes31@gmail.com \\ Antônio Eduardo de Oliveira Silva \\ ORCID: https://orcid.org/0000-0002-7149-7152 \\ Universidade Potiguar, Brasil \\ E-mail: eduardohiuga20100@gmail.com \\ Livia Kallahan Soares Pequeno \\ ORCID: https://orcid.org/0000-0001-5453-9245 \\ Universidade Potiguar, Brasil \\ E-mail: liviakallahan@gmail.com \\ Bruna Grandi Fernandes \\ ORCID: https://orcid.org/0000-0003-3803-361X \\ Universidade Potiguar, Brasil \\ E-mail: brunagrandi85@gmail.com \\ Ramile Cristyan Dos Santos \\ ORCID: https://orcid.org/0000-0001-8012-0640 \\ Universidade Potiguar, Brasil \\ E-mail: rcristyans@gmail.com \\ Alycia Dannyela de Lima Silva Gobbi \\ ORCID: https://orcid.org/0000-0002-2627-1314 \\ Universidade Potiguar, Brasil \\ E-mail: alyciadannyela.al@gmail.com \\ Marcília Ingrid Lima Barroso Nunes \\ ORCID: https://orcid.org/0000-0002-5871-3930 \\ Universidade Potiguar, Brasil \\ Universidade do Estado do Rio Grande do Norte, Brasil \\ E-mail: prof.marciliabarroso@gmail.com \\ Raille Silva de Jesus \\ ORCID: https://orcid.org/0000-0002-8236-6588 \\ Universidade Potiguar, Brasil \\ E-mail: silvaraille@gmail.com \\ Ana Camila Campelo de Albuquerque Nunes \\ ORCID: https://orcid.org/0000-0002-2030-8331 \\ Universidade Potiguar, Brasil \\ Centro Universitário Natalense, Brasil \\ E-mail: ccampelo32@gmail.com
}

\begin{abstract}
Resumo
Em virtude do isolamento social ocasionado pela pandemia da COVID-19, as realizações de atividades físicas passaram por adaptações necessárias através de plataformas digitais de comunicação interativa. Para que a prática esportiva fosse possível, os profissionais de educação utilizaram-se de recursos para driblar as dificuldades. O objetivo deste estudo é mostrar como os profissionais de educação física atuaram durante a pandemia de COVID-19 e as ferramentas e plataformas de atuação utilizadas. O trabalho tem como objetivos específicos; a) conhecer quais as formas que os profissionais de educação física encontraram de obter novos resultados; b) mostrar como o profissional de educação física utilizou as plataformas digitais nesse período de pandemia; c) verificar qual melhor forma de obter resultados por
\end{abstract}


meio da internet. Trata-se de uma pesquisa de análise descritiva, quantitativa de caráter transversal, através da aplicação de um questionário de elaboração própria. A amostra da pesquisa foi composta por profissionais de educação física formação plena ou bacharelado. Os resultados obtidos mostram que a Internet foi um meio eficaz para manter a periodicidade dos treinamentos, ajudando assim, no enfrentamento da pandemia do COVID-19. Conclui-se que é de fundamental importância que os profissionais de educação física possam reinventar-se e superar os desafios impostos pelo mercado.

Palavras-chave: Atividade física; COVID-19; Educação física e treinamento; Internet.

\begin{abstract}
Due to the social isolation caused by the COVID-19 pandemic, the performance of physical activities underwent necessary adaptations through digital platforms of interactive communication. For the practice to be possible, education professionals used resources to overcome the difficulties. The aim of this study is to show how physical education professionals acted during the COVID-19 pandemic and the tools and platforms used. The work has as specific objectives; a) to know the ways physical education professionals have found to obtain new results; b) to show how physical education professionals used digital platforms during this pandemic period; c) to check best to get results through the internet. This is a descriptive analysis, quantitative cross-sectional research, through the application of a questionnaire designed in-house. The research sample consisted of physical education professionals with full training or bachelor's degree. The results obtained show that the use of the Internet was an effective way to maintain the frequency of training, thus helping to fight the COVID-19 pandemic. It is concluded that it is of fundamental importance that physical education professionals can reinvent themselves and overcome the challenges imposed by the market.
\end{abstract}

Keywords: Physical activity; COVID-19; Physical education and training; Internet.

\title{
Resumen
}

Debido al aislamiento social provocado por la pandemia COVID-19, la realización de actividades físicas sufrió las adaptaciones necesarias a través de plataformas digitales de comunicación interactiva. Para que la práctica del deporte fuera posible, los profesionales de la educación utilizaron recursos para superar las dificultades. El objetivo de este estudio es mostrar cómo actuaron los profesionales de la educación física durante la pandemia del covid-19 y las herramientas y plataformas utilizadas. El trabajo tiene como objetivos específicos; a) conocer qué caminos han encontrado los profesionales de la educación física para obtener nuevos resultados; b) mostrar cómo los profesionales de la educación física utilizaron las plataformas digitales durante este período pandémico; c) comprobar la mejor forma de obtener resultados a través de Internet. Se trata de una investigación descriptiva, cuantitativa, transversal, mediante la aplicación de un cuestionario de diseño propio. La muestra de investigación estuvo constituida por profesionales de la educación física con formación completa o licenciatura. Los resultados obtenidos muestran que Internet fue un medio eficaz para mantener la frecuencia de los entrenamientos, contribuyendo así a combatir la pandemia Covid-19. Se concluye que es de fundamental importancia que los profesionales de la educación física puedan reinventarse y superar los desafíos que impone el mercado.

Palabras clave: Actividad física; COVID-19; Educación y entrenamiento físico; Internet.

\section{Introdução}

Em 11 de março de 2020 a Organização Mundial da Saúde (OMS) declarou que o surto de COVID-19 tinha evoluído para uma pandemia (Hien Lau et al., 2020). A COVID-19 é uma doença infecciosa e contagiosa oriunda do Coronavírus que desencadeia uma síndrome respiratória aguda grave 2 (SARS-CoV-2). É uma infecção respiratória conhecida desde a década de 60 (Meyer\&Velavan, 2020) e sua abreviação em do inglês: Corona Vírus Disease 2019.

Para o controle epidemiológico, centenas de países adotaram medidas de distanciamento social no qual proibiram situações de aglomerações de pessoas, tanto em locais públicos quanto em locais privados, com proibições de festas e eventos, funcionamento de academias e clubes esportivos, escolas, mercado e entre outros segmentos, recomendando que a população ficasse em casa o máximo de tempo possível, criando medidas para impedir novos casos, e contendo o avanço da doença, ou até mesmo lockdown para reduzir a transmissão do vírus (Escher, 2020).

Durante o pico da pandemia, foram adotadas estratégias que apesar de necessárias, foram preocupantes, pois isso iria reduzir os níveis de atividades físicas na população (Harmer et al., 2020). Essa redução causaria sedentarismo e resultaria na perda de condições físicas, na saúde e na funcionalidade (Barbalho et al., 2017). Uma das estratégias conhecida como nãofarmacológica é a prática de exercício físico em que evitaria e proporcionaria a prevenção de diversas doenças, sejam elas físicas, psicológicas ou de caráter metabólicos (Luan et al., 2019). 
Segundo Turner e Campbell (2018) vale destacar ainda a ação dos exercícios físicos no sistema imunológico. É sugerido que a prática de exercício físico é capaz de fortalecer o sistema imunológico, inclusive diminuindo a incidência de doenças transmissíveis como as infecções virais (WU et al., 2020), tais como o novo coronavírus (SARS-CoV-2), causador da COVID19.

Os profissionais de educação física despertaram o interesse em aprofundar seus conhecimentos, nessas novas plataformas para poderem trabalhar com clientes que requer-um pouco mais de cuidados por algum motivo. Os profissionais passaram a lecionar aulas de forma remota, para os alunos que não podiam ir pessoalmente, nomeando-as de aulas virtuais, passaram a realizar o atendimento e acompanhamento das atividades físicas dos alunos de forma remota, adaptando os treinos a nova rotina e ambiente no qual aquele individuo iria estar inserido, com videoconferências e lives em redes sociais. Dessa forma, a importância do hábito da prática de atividades físicas para a saúde continuou sendo preservada e evidenciada.

Para os professores, o grande desafio é a adaptação ao novo meio que requer mudanças na forma de produzir e repassar conteúdo. Para isso, necessita-se evoluir, utilizando essas novas ferramentas que facilitam a comunicação, para que possam manter suas aulas dinâmicas.

\subsection{Objetivo Geral}

Analisar as modificações de trabalho no cenário do profissional de Educação Física Bacharelado ou Pleno durante a pandemia de COVID-19 e o isolamento social.

\subsubsection{Objetivos Específicos}

a) Conhecer quais as formas que os Profissionais de Educação Física encontraram de obter novos resultados.

b) Mostrar como o Profissional de Educação Física utilizou as plataformas digitais nesse período de pandemia de COVID-19.

c) Verificar qual melhor forma de obter resultados por meio da Internet.

\subsection{Justificativa}

No viés acadêmico, considerando-se que proporcionará o aporte de dados, informações e achados científicos atualizados a respeito da referida temática, possibilitando que os graduandos e graduados aportem conhecimento e, por vias disso, promovam o desenvolvimento acadêmico da sociedade como um todo. Além de mostrar à população que os profissionais de Educação Física podem atuar de outras formas, como aulas remotas sendo orientada pelo profissional e a prática de exercícios em qualquer lugar.

Espera-se que outras pessoas possam usufruir desse material e agregar conhecimento. Assim, observou-se a necessidade de mostrar o quanto a área de Educação Física passou por modificações durante a pandemia e que os profissionais tiveram de se reinventar-se e conhecer mais sobre a COVID-19.

\section{Revisão de literatura}

\subsection{Educação Física Bacharel}

No processo de reestruturação surgiu a possibilidade da habilitação para o profissional de Educação Física, o bacharelado, que permitiu novos olhares para a prática profissional na área, com uma nova percepção e concepção do significado da prática profissional. Antigamente a prática no contexto da preparação profissional era pensada e desenvolvida para o contexto 
escolar. Foi então que com o bacharelado a prática foi conduzida para ambientes como as academias, clubes, empresas de lazer, e hospitais (Oliveira, 2011, p.137).

A formação do Bacharel em Educação Física concentra-se na área não escolar e tem o objetivo de formar um profissional nos princípios da flexibilidade curricular, capaz de acompanhar as demandas e tendências de um mercado dinâmico e globalizado, para atuar em: Instituições e Órgãos de Administração e Prática Desportiva; Clubes; Associações Desportivas e Recreativas; Centros de Recreação e Lazer; Academias; Centros e Laboratórios de Pesquisa; Hotéis; Campings; Condomínios; Centros de Estética; Instituições e Órgãos de Saúde; Clínicas; Centros de Saúde; Hospitais; Empresas e Instituições públicas e Privadas e como Autônomos (Oliveira, 2011, p. 137).

Historicamente a verdade é que essas práticas já ocorriam mesmo sem uma formação acadêmica, as academias, os clubes, entre outros tinham em seus quadros, profissionais práticos que muitas vezes não eram formados, surgi daí a necessidade de formação de profissionais para atuar no mercado. Assim, a resolução 03/87 possibilitou um novo olhar para o processo de preparação do profissional em Educação Física além do ambiente escolar, fato muito importante para a categoria (Tojal, 1991).

Cabe agora à universidade e à Educação Física a missão de desenvolver estudos e organizar o conhecimento, visando oferecer a sociedade respostas satisfatórias que venham a tender a seus anseios pela recreação e lazer, pelo treino e manutenção de boa condição física, pelo atendimento à pessoa portadora de deficiência, pelo desenvolvimento de uma cultura de vida saudável e tantos outros aspectos que merecem ser satisfeitos (Tojal, 1991).

Em primeiro de setembro de 1998, se deu-se a regulamentação do profissional efetivada de acordo com a Lei 9.696, com a criação do Conselho Federal de Educação Física (CONFEF) junto de seus respectivos Conselho Regional de Educação Física (CREF) em suas regiões do país. CONFEF é uma instituição de direito público com o objetivo de disciplinar, orientar e fiscalizar o exercício das atividades exercidas por profissionais de Educação Física. Por ser constituído um órgão orientador influenciou e ainda influencia várias resoluções. Isso tudo já acontecia em 2002.

Até o ano de 1987, a formação profissional era regida pela Resolução 69/69 que regulamentava o curso de licenciatura em Educação Física e Técnico de Desportos, a partir desse ano, foi aprovada a Resolução 03/87, sua importância é fundamental pois as linhas gerais foram mantidas para a reformulação da nova proposta de diretrizes para a preparação profissional em Educação Física.

As atuais diretrizes curriculares (Resolução 07/2004) respeitam um dos aspectos preconizados pela Lei de Diretrizes em Bases da Educação Nacional (LDB) e, assim permite a continuidade a um dos aspectos considerados fundamentais e evolutivos da Resolução 03/1987, que é a autonomia e a flexibilidade. As Diretrizes Curriculares para os cursos de graduação em Educação Física foram desenvolvidas de modo a assegurar a indissociabilidade teoria-prática. Os termos teoria e prática são apresentados como distintos e com significados diferente, porém complementares.

Um dos pontos fundamentais no atual processo de preparação profissional de Educação Física que deve ser analisado com profundidade a partir das reflexões apresentadas pela resolução 03/87 é a relação teoria e prática. A análise requer um olhar atento sobre a organização dos conteúdos curriculares, práticas pedagógicas e estágios curriculares no processo de preparação. Assim compreende-se que a Educação Física possui uma longa trajetória, geradora de conhecimentos práticos e teóricos, que são de grande importância para população.

\subsection{Pandemia e isolamento social}

Segundo Lewnard e Lo (2020) por consequências do número de óbitos, foi criado a estratégia do isolamento e distanciamento social tentando reduzir o contágio da COVID-19. O isolamento social afetou todos, inclusive as academias de 
ginásticas, box de cross training, clubes esportivos, além de espaços fitness em condomínios, obrigando a população a ficar em casa, evitando aglomerações.

Segundo especialistas em saúde pública, as academias eram de fato um lugar propicio para transmissão do coronavírus, por ser transmitido por gotículas de saliva no ar ou de superfícies infectadas, pois o ato de pegar e passar a mão no rosto expõe o indivíduo ao vírus. Gerando assim, dificuldade para realizar os exercícios físicos e seguirem com suas rotinas, em virtude da população em confinamento (Andrade\&Liz, 2016).

\subsection{Importância da atividade física e do profissional de Educação Física na prática de atividade física durante a pandemia}

Existem recomendações de várias entidades que incentivam a prática de exercício em casa, sendo já de conhecimento e embasamento científico na literatura. Os exercícios resistidos ou manuais são ótimos, além do uso de faixas elásticas. O Decreto federal 10.344, de 11 de maio de 2020 reconheceu a prática de exercícios físicos para a prevenção e promoção da saúde e incluif as academias no rol das atividades essenciais na pandemia, garantindo seu funcionamento, seguindo alguns protocolos.

No contexto atual, vê-se a importância da Educação Física na área da saúde, pois esta visa tanto a recreação, como reabilitação e, principalmente, a prevenção e promoção da saúde, além da educação do corpo para que ocorra a preservação. Coube então à Educação Física a responsabilidade de orientar as pessoas promovendo saúde, ensinando-lhes a ter qualidade de vida através de exercícios físico, de caminhadas, exercícios que também podem ser feitos até mesmo em sua própria residência, atividades recreativas, culturais e desportivas também, existem muitas variedades e formas. Ficando assim clara sobre diversas ações de prevenção que podem ser feitas por meio da educação, saúde e justiça (Ferraz, 2000).

Atualmente a Educação Física está passando por vários desafios para manter-se com suas aulas durante a pandemia de COVID-19, tendo que se reinventar com novos perfis e fazer bastante o uso da internet para divulgar seu trabalho. O profissional de Educação Física encontra-se em volta de grandes desafios. Crescem as demandas do mercado, aumenta a tensão entre o acesso, a qualidade e a concorrência entre eles, nas redes sociais.

Segundo Dias et al. (2007) A importância desse profissional pode ser avaliada pelo programa de exercício mal elaborado que pode causar várias consequências, uma delas é a hipoglicemia, a perda de proteínas ao urinar, complicações mais graves como taque cardíaco ou morte súbita, principalmente se for hipertenso ou diabético. Deve-se observar e ter conhecimento do histórico de saúde para que não passe do limite, trabalhar para obter vantagens do exercício, pois se trabalhar ao contrário a uma grande probabilidade de riscos do que de benefícios.

\subsection{Como os profissionais de Educação Física estão atuando no novo cenário}

Segundo o Colégio Americano de Medicina do Esporte, é recomendado a prática de exercício físicos para todos, sendo do grupo de risco ou não, com o objetivo de melhorar a função imunológica, diminuir ansiedade e o estresse percebido. Essa recomendação se estende a pessoas em distanciamento social que não estejam infectadas pelo SARS-CoV-2 e para pessoas que estejam infectadas, mas permanecem assintomáticas (Joy, 2020).

Um dos desafios para as práticas de exercício físicos nesse tempo de pandemia é justamente estimular os praticantes a compreender que esse espaço tecnológico também é um espaço de construção de conhecimento coletivo. Produzir conhecimento juntamente com os alunos dentro desse formato de aula à distância fazendo com que se envolvam com a atividade física e ao mesmo tempo estimular a prática de exercício. Como exemplo investirem em aulas online, podendo ser gravadas uma sequência de exercícios, explicando com exatidão a execução de cada movimento. Outra possibilidade é o atendimento em tempo real, no qual a orientação ocorre quando é feita a atividade (Oliveira et al., 2020).

Em relação a intensidade de exercícios moderados, Jiménez-Pavón, Carbonell-Baeza \& Lavie (2020), a recomendam que os exercícios sejam de intensidade e volume moderados, evitando a utilização de altas intensidades concomitantemente de 
alto volume (exercícios extenuantes), pois grande quantidade de exercício pode chegar imunodepressão, por isso a importância de um acompanhamento com um profissional capacitado, para proporcionar melhor orientação sobre o planejamento do programa de treinamento. Pesquisas evidenciam que o exercício sendo realizado de forma correta e controlada (periodização) surtido efeitos, proporcionando melhoras nas funções imunes, auxiliando na resposta imunológica através do exercício o organismo ficara reativo a doenças e pronto para enfrentar e vencer doenças como coronavírus.

Vale ressaltar que os profissionais de Educação Física estão reinventando-se e utilizando de redes sociais para divulgar e mostrar seu trabalho, possibilitando desenvolver habilidades, ao formato digital, onde a demanda aumenta cada dia mais por rotinas saudáveis (Oliveira et al., 2020).

\section{Metodologia}

\subsection{Tipo de Pesquisa}

Esta é uma pesquisa de análise descritiva, quantitativa de caráter transversal, por tratar-se de uma coleta que ocorreu em um determinado instante de tempo e trazer apenas análises descritivas de um determinado tema (Simões, 2009).

\subsection{População e Amostra de Voluntários}

A amostra da pesquisa foi composta por profissionais de Educação Física bacharelado ou da formação plena, totalizou

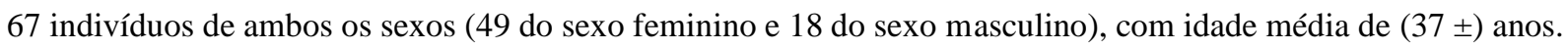

\subsection{Instrumento para Coleta dos Dados}

Foi utilizado um questionário de criação própria dos pesquisadores sendo adaptado para caracterizar as perguntas de acordo com o processo da pandemia.

\subsection{Procedimentos de Coleta de Dados}

A coleta foi realizada de forma virtual durante o período do mês de abril de 2021, por meio de questionário eletrônico via Plataforma Google Forms. Os questionários foram aplicados de forma eletrônica no mês de abril de 2021, com indivíduos residentes no estado do Rio Grande do Norte. A resolução dos questionários ocorreu de forma individual e on-line devido ao processo de pandemia.

\subsection{Critérios de Inclusão e Exclusão}

Como critérios inclusão:

- Os participantes deveriam ser residentes do município de Natal

- Possuírem formação em Educação Física Bacharelado e Educação Física Plena

- Além da formação devem atuar na área.

Os critérios de exclusão avaliados dessa pesquisa, é ainda estarem em processo de formação na faculdade, não serem formados em bacharel, não assinarem o termo de consentimento on-line ou não responderem a pelo menos uma das questões do questionário eletrônico.

\subsection{Procedimentos Estatísticos}

Foi utilizado o Excel 2016 para tratamento dos dados, realização de gráficos e porcentagens das variáveis quantitativas e qualitativas. 


\section{Resultados e Discussões}

De acordo com o acompanhamento realizado pela empresa In Loco (2020), o percentual de distanciamento no Rio Grande do Norte chegou a uma média de $70 \%$ no início do pico da pandemia, ainda em março, mas esse percentual vem diminuindo - e preocupando cada vez mais. A partir dos dados coletados nesta pesquisa onde será destacado os principais achados de forma descritiva e realizar uma análise das modificações de trabalho dos profissionais de Educação Física, bacharelado ou pleno, durante a pandemia de COVID-19 e o isolamento social, apontando como eles se reinventaram nesse processo.

\subsection{Análise geral dos profissionais desta pesquisa e os impactos da pandemia COVID-19 em seu trabalho}

O Profissional de Educação Física é especialista em atividades físicas, nas suas diversas manifestações - ginásticas, exercícios físicos, desportos, jogos, lutas, capoeira, artes marciais, danças, atividades rítmicas, expressivas e acrobáticas, musculação, lazer, recreação, reabilitação, ergonomia, relaxamento corporal, ioga, exercícios compensatórios à atividade laboral e do cotidiano e outras práticas corporais -, tendo como propósito prestar serviços que favoreçam o desenvolvimento da educação e da saúde, contribuindo para a capacitação e/ ou restabelecimento de níveis adequados de desempenho e condicionamento fisiocorporal dos seus beneficiários, visando à consecução do bem-estar e da qualidade de vida, da consciência, da expressão e estética do movimento, da prevenção de doenças, de acidentes, de problemas posturais, da compensação de distúrbios funcionais, contribuindo ainda, para consecução da autonomia, da autoestima, da cooperação, da solidariedade, da integração, da cidadania, das relações sociais e a preservação do meio ambiente, observados os preceitos de responsabilidade, segurança, qualidade técnica e ética no atendimento individual e coletivo (CONFEF, 2002, p. 134).

Conforme dados da referida pesquisa, o maior percentual de profissionais atua em academias. Acordante o Gráfico 1, 42 profissionais (63\%) trabalham em academias, $16(25 \%)$ realizam atendimento domiciliar e 9 (12\%) em clubes.

Gráfico 1. Área de atuação dos profissionais de Educação Física.

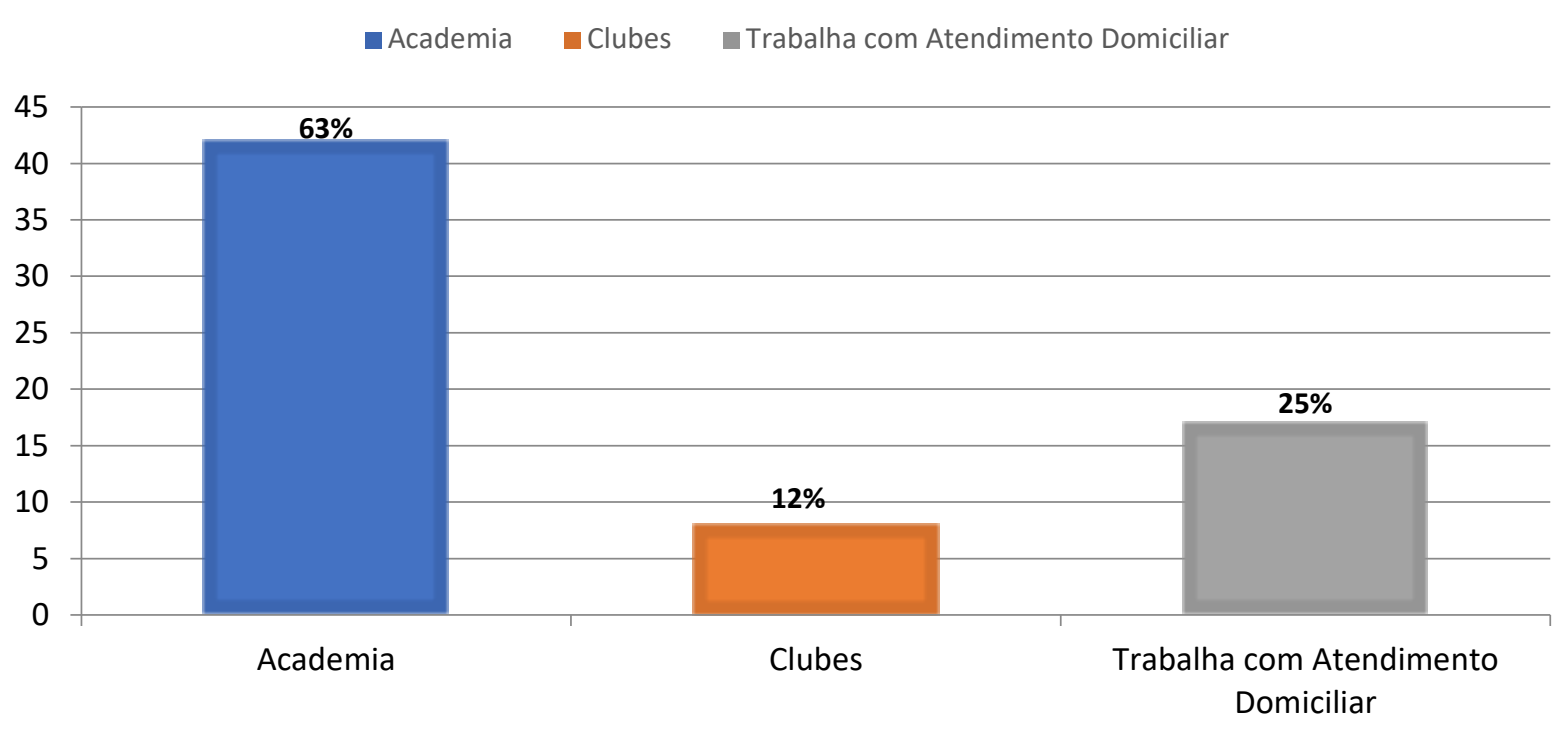

Fonte: Dados da pesquisa.

Em relação a continuidade do trabalho destes profissionais, o Gráfico 2 aponta que 40 profissionais (60\%) trabalharam durante a pandemia e 27 (40\%) não trabalharam. Porém, mesmo esses dados apontando uma continuidade no trabalho desses profissionais, ele não anula a diminuição de alunos e consequentemente ocorre um abalo financeiro em suas rendas. 
De acordo com dados da Organização para a Cooperação e Desenvolvimento Econômico (OCDE, 2020), os impactos da pandemia de COVID-19 sobre o cenário econômico podem ser vistos no seu pior momento, proporcionando que o crescimento econômico mundial caia pela metade, impactando diretamente todos os setores da economia.

Gráfico 2. Atuação durante a pandemia dos profissionais de Educação Física.

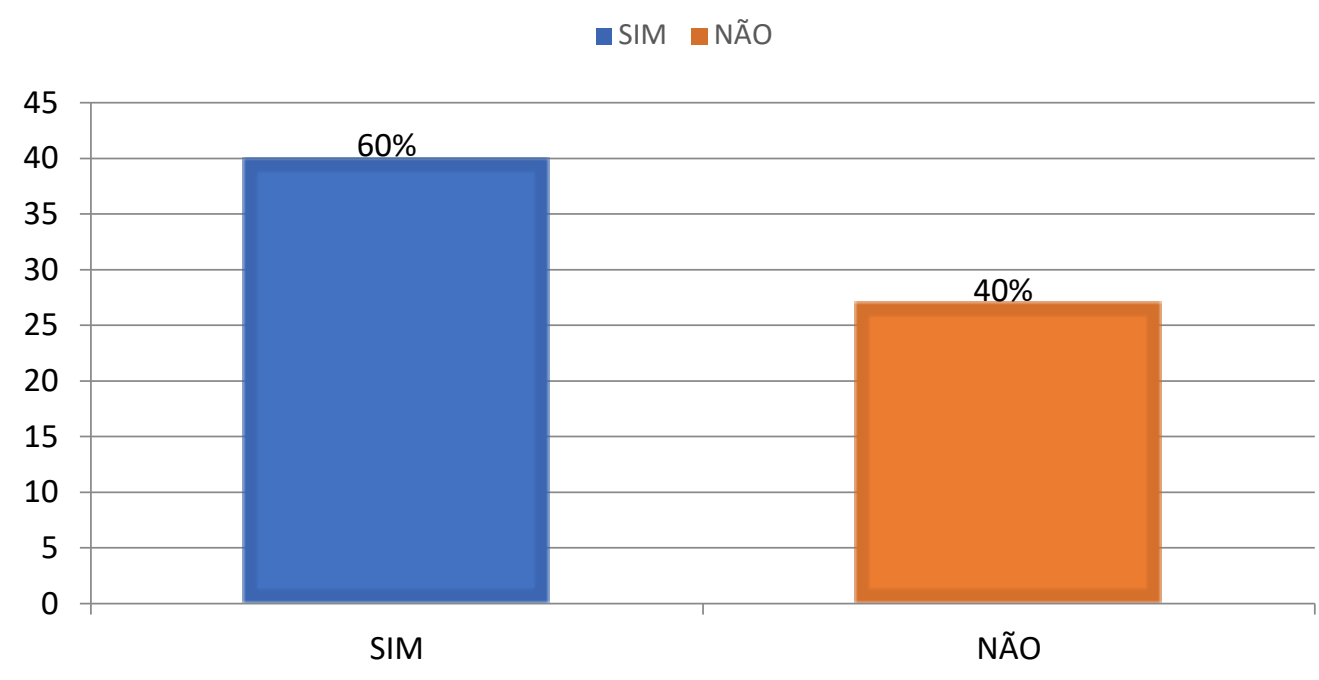

Fonte: Dados da pesquisa.

O caso dos profissionais que trabalham com o treinamento personalizado (personal trainer), que são a grande parte dos voluntários desta pesquisa, tem sentido instabilidade em seus atendimentos com o distanciamento social e o fechamento das academias e dos centros de realização de exercícios físicos como aponta o gráfico 3 e 4. Neste gráfico, 60 profissionais (90\%) sofreu impactos em seu trabalho, sendo que 5 (7\%) não sofreu alterações e 2 (3\%) responderam outros.

Gráfico 3. A pandemia do COVID-19 afetou o trabalho dos profissionais de Educação Física.

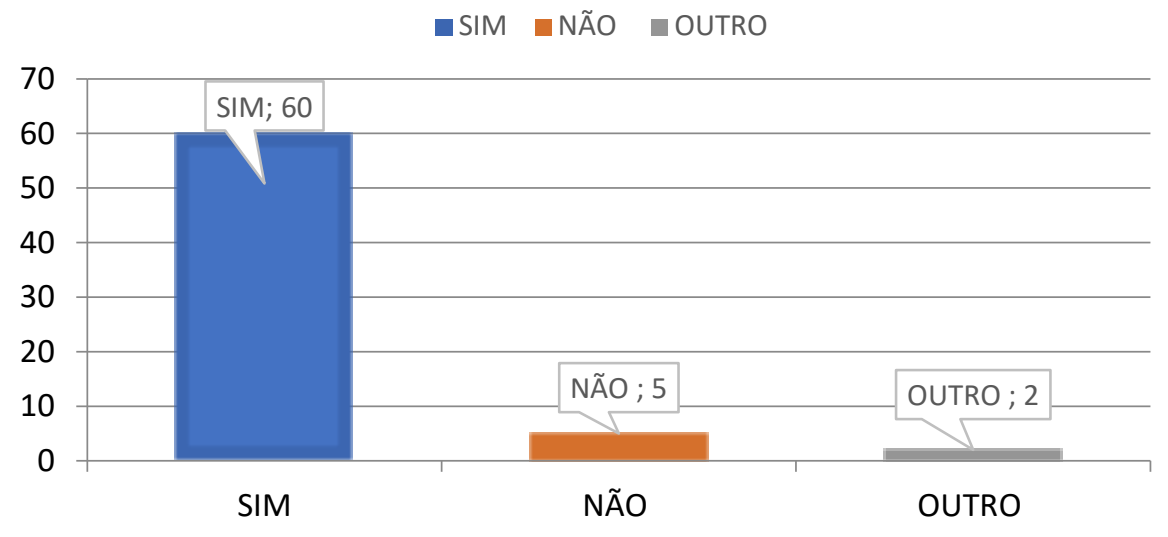

Fonte: Dados da pesquisa.

Em relação ao Gráfico 4, é possível perceber alterações em seus expedientes de trabalho, porém, de forma positiva. Dos participantes, 42 (63\%) tiveram alterações, $10(15 \%)$ mantiveram-se estáveis, 5 (7\%) diminuíram sua carga de horário e $10(15 \%)$ responderam outros.

Na literatura, é possível observar os efeitos macroeconômicos da epidemia SARS em (2003), proporcionando quedas significativas nas economias, devido à redução no consumo de serviços e bens, o que acarretou um aumento de custos 
operacionais de negócios e avaliação sobre os riscos dos países. Quanto maior a suscetibilidade à doença, maiores foram os níveis de choque econômico, onde os custos globais foram significativos e não se limitaram exclusivamente aos países afetados de forma direta (MCkibbin, 2020).

Gráfico 4. Carga horária dos profissionais de Educação Física durante a pandemia de COVID-19.

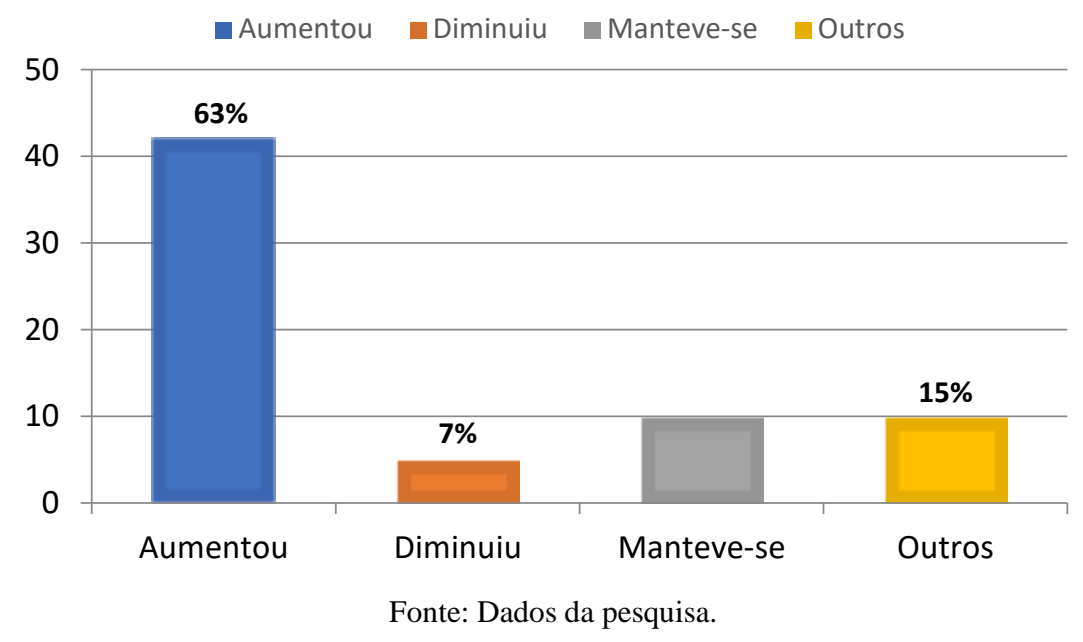

4.2 Análise geral dos profissionais desta pesquisa e o processo de reinvenção de seu trabalho devido a pandemia COVID-

19

A pandemia de COVID-19 trouxe impactos significativos na vida dos indivíduos em nível global, chamando à atenção a abrangência e velocidade com a qual disseminou-se. Alguns dados históricos, embora ainda muito recentes para uma análise rigorosa, revelam essa dinâmica espaço-temporal da doença (OMS, 2020). Para diminuir o impacto apontado acima, os profissionais de Educação Física precisaram reinventar-se e se adaptar-se, possibilitando a continuidade de suas atividades laborais e evitando da prática de exercícios físicos pela população.

O Gráfico 5 denota que, a maioria dos profissionais adequaram-se a pandemia, com uma amostra de 51 destes (76\%) reinventando-se para alcançar seu público alvo de trabalho, principalmente aqueles que trabalham com grupos especiais, em contra partida, são 12 profissionais (18\%) dos não procuraram mudanças e outros 4 (6\%) optaram por outros meios. Como relatado anteriormente, o vírus tem alta proporção de contaminação e um alto grau de mortalidade, especialmente entre indivíduos acima de 60 anos, pacientes oncológicos, pessoas que possuem doenças autoimunes, portadores de HIV, transplantados, diabéticos e hipertensos. Ou seja, há um enorme grupo de risco que necessita de uma atenção ainda mais especial pelos órgão da saúde, fazendo com que a ordem primária para evitar o contágio seja o isolamento social em forma de quarentena, utilizando os serviços externos com a menor frequência possível ou no caso de comprovada necessidade (Fang et al., 2020). 
Gráfico 5. Ressignificação dos profissionais de educação física durante a pandemia de COVID-19.

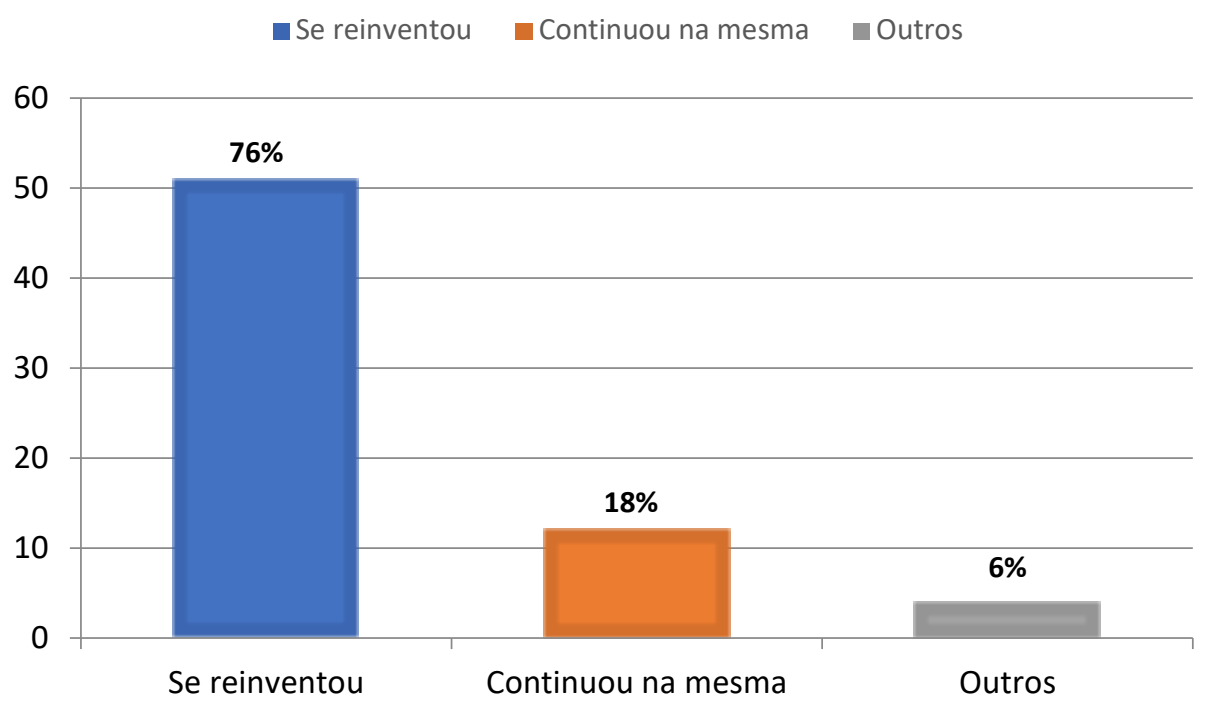

Fonte: Dados da pesquisa.

Para que seus alunos não parassem de realizar atividades físicas díaria, seja alunos de grupo de risco ou não, a grande maioria dos profissionais desta pesquisa utilizaram a Internet como plataforma de trabalho, como mostra o Gáfico 6, onde 52 profissionais $(78 \%)$ da amostra fizeram uso da plataforma, $13(19 \%)$ não utilizaram e 2 (3\%) optaram por outras formas. Outro ponto a ser considerado é a periodicidade da atividade física como meio de promoção da saúde e qualidade de vida, promovendo melhora fisiológica (controle da glicose, melhor qualidade do sono, melhora da capacidade física relacionada à saúde); psicológica (relaxamento, redução dos níveis de ansiedade e estresse, melhora do estado de espírito, melhoras cognitivas) e social (indivíduos mais seguros, e cultural ampliada, entre outros); além de prevenir algumas doenças (Salin et al., 2011; Nahas, 2001).

Gráfico 6. Utilização da internet como plataforma de trabalho.

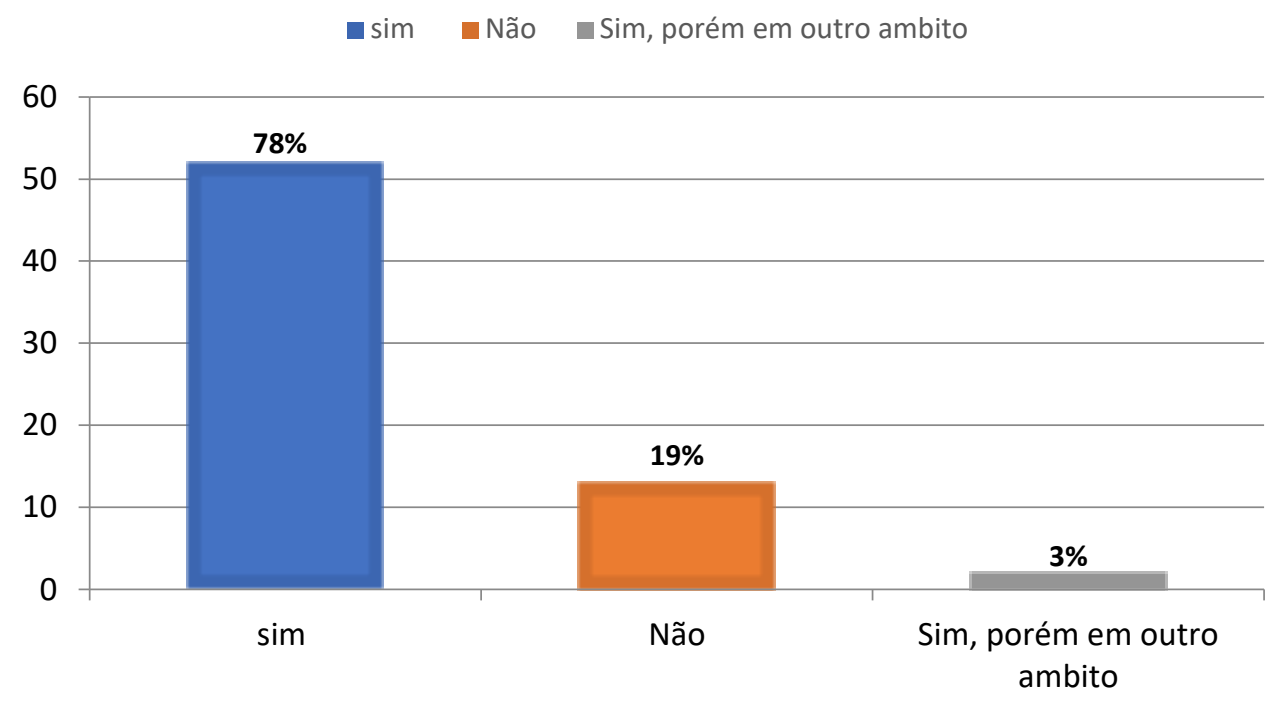

Fonte: Dados da pesquisa.

A utilização da forma remota para os treinos diário, como mostra o Gráfico 7, foram mais aplicados em forma de vídeo aula. Dos voluntários, 23 (34\%) profissionais utilizaram vídeo 14 (24\%) utilizaram aulas ao vivo, 8 (12\%) em forma de aplicativo e $22(33 \%)$ usaram outra forma de trabalho. 
Gráfico 7. Formas de aplicação do trabalho de forma remota.

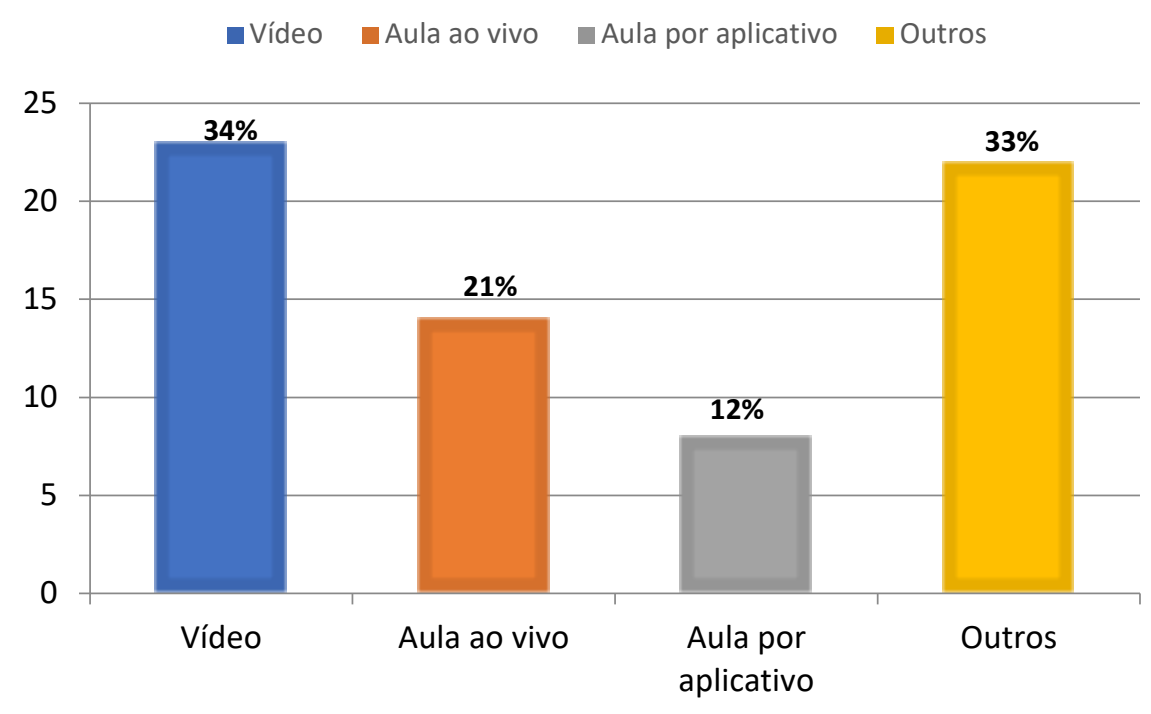

Fonte: Dados da pesquisa.

Em relação ao treino, 44 (66\%) dos profissionais de Educação Física apontam (Gráfico 8), eficácia em treinos realizados em casa e outros $18(27 \%)$ profissionais que não tiveram a mesma eficacia, e 5 (7\%) deles, responderam outros.

Gráfico 8. Eficácia do treino de forma remota.

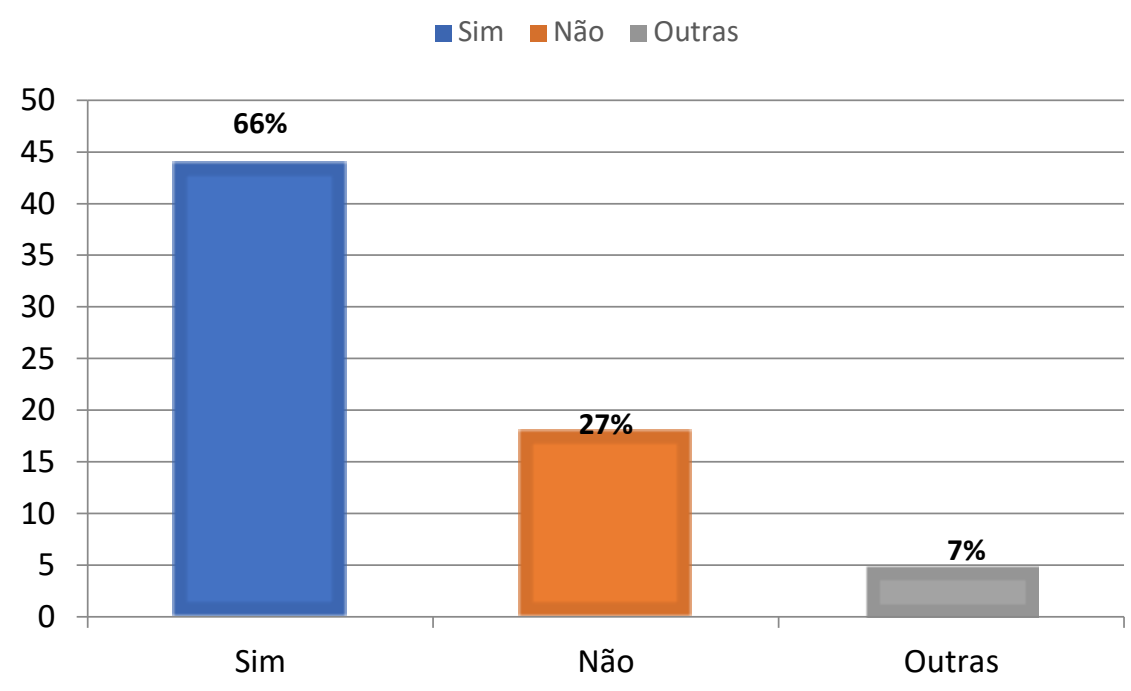

Fonte: Dados da pesquisa.

Sobre essa temática, de forma específica, não há estudos corroborando ou não com nossos achados, porém estudo como o de Neto et al. (2020) aponta-que é um desafio, adaptar a prescrição do treinamento a ser realizado em casa. Nesse sentido, o autor apresenta duas grandes barreiras a serem enfrentadas nesse processo: (a) não há uma variedade de equipamentos disponíveis para treinamento, (b) o treinamento deve ser realizado à distância, o que dificulta o controle das variáveis.

Ainda sobre a temática, os autores apontam que para realizar o exercício aeróbico no ambiente doméstico, há muitas possibilidades, como marcha estacionária ou subir e descer escadas e que, o uso de ergômetros domésticos, também se torna uma opção para quem possui este tipo de equipamento. Exercícios popularmente conhecidos como polichinelos, pular corda, entre outros, também são opções, desde que a condição musculoesquelética do praticante permita (Neto et al., 2020). Essas 
atividades podem ser realizadas continuamente (por exemplo, 30 minutos contínuos) ou em blocos de 2, 5, 10 e 15 minutos, desde que o total acumulado na semana seja $\geq 150$ minutos, conforme sugerido pelo ACSM (Joy, 2020; Garber et al., 2021)

\section{Conclusão}

Analisando os achados extraídos do estudo, percebe-se que os profissionais de Educação Física ressignificaram seu trabalho, adaptando-se ao mercado e possibilitando que seus alunos não suspendessem suas práticas de atividades físicas diárias.

Diante desse quadro de pandemia de COVID-19, é necessário o melhor entendimento de como a estratégia desses profissionais são importantes não só no cunho trabalhista, como também de estratégia para a prática aplicação de treino nessas condições atuais que impactam de forma significativa na vida das pessoas. Assim, também, investigar diferentes formas de ação para que o isolamento afete menos o bem-estar e a saúde física das pessoas

Sugere-se mais investigações acerca de como a pandemia de COVID-19 afetou a rotina trabalhista dos profissionais de Educação Física a fim de obter dados que auxiliem na elaboração de alternativas de prevenção para futuras situações adversas como esta.

\section{Referências}

Brasil. (1987). Resolução n. ${ }^{\circ}$ 03/87, de 16 de junho de 1987. Fixa os mínimos de conteúdo e duração a serem observados nos cursos de graduação em Educação Física (Bacharelado e/ou Licenciatura Plena). Diário Oficial da República Federativa do Brasil, Brasília, DF.

Brasil. (2004) Resolução nº 07, de 31 de março de 2004. Diretrizes Curriculares Nacionais para os cursos de graduação em Educação Física, em nível superior de graduação plena. Conselho Nacional da Educação, Brasília. https://periodicos.uem.br/ojs/index.php/EspacoAcademico/article/view/15750/9570.

Barbalho, M. S. M., Gentil, P., Izquierdo, M., Fisher, J., Steele, J., \& Raiol, R. A. (2017). There are no no-responders to low or high resistance training volumes among older women. Experimental Gerontology, 99, 18-26. https://www.sciencedirect.com/science/article/abs/pii/S0531556517304874. https://doi.org/10.1016/j.exger.2017.09.003.

Chen, P., Mao, L., Nassis, G. P., Harmer, P., Ainsworth, B. E. \& Li, F. (2020). Coronavirus disease (COVID-19): The need to maintain regular physical activity while taking precautions. Journal of Sport and Health Science, 9(2), 103-04. https://www.ncbi.nlm.nih.gov/pmc/articles/PMC7031771/. 10.1016/j.jshs.2020.02.001.

Campbell, J. P. \& Turner, J. E. (2018). Debunking the Myth of Exercise-Induced Immune Suppression: Redefining the Impact of Exercise on Immunological Health Across the Lifespan. Frontiers in Immunology, 9, 648, abr. 2018. https://pubmed.ncbi.nlm.nih.gov/29713319/. https://doi.org/10.3389/fimmu.2018.00648.

Dias, J A. et al. (2007). A importância da execução de atividade física orientada: uma alternativa para o controle de doença crônica na atenção primária. Revista Digital EFDeportes.com, Buenos Aires, 12(114). https://www.efdeportes.com/efd114/a-importancia-da-execucao-de-atividade-fisica-orientada.htm.

Escher Jr, A. R. (2020). An Ounce of Prevention: Coronavirus (COVID-19) and Mass Gatherings. Cureus, 12(3), e7345. https://www.ncbi.nlm.nih.gov/pmc/articles/PMC7089620/pdf/cureus-0012-00000007345.pdf. DOI: 10.7759/cureus.7345.

Fang, L., Karakiulakis, G., \& Roth, M. (2020). Are patients with hypertension and diabetes mellitus at increased risk for COVID-19 infection?. The Lancet Respiratory Medicine, 8(4), e21. https://www.thelancet.com/journals/lanres/article/PIIS2213-2600(20)30116-8/fulltext. DOI: https://doi.org/10.1016.

Garber, C. E. et al. (2018). Quantity and quality of exercise for developing and maintaining cardiorespiratory, musculoskeletal, and neuromotor fitness in apparently healthy adults. Medicine \& Science in Sports \& Exercise, 43(7), 1334-59. https://journals.lww.com/acsmmsse/Fulltext/2011/07000/Quantity_and_Quality_of_Exercise_for_Developing.26.aspx. DOI: 10.1249/MSS.0b013e318213fefb.

Jiménez-Pavón, D., Carbonell-Baeza, A. \& Lavie, C. J. (2020). Physical exercise as therapy to fight against the mental and physical consequences of COVID19 quarantine: Special focus in older people. Elsevier, 63(3), p. 386-88. https://www.sciencedirect.com/science/arti cle/abs/pii/S0033062020300633?via\%3Dihub. DOI: https://doi.org/10.1016/j.pcad.2020.03.009.

Joy, Liz et al. (2020). Staying active during COVID-19. American College of Sports Medicine.

Lau, H. et al. (2020). Internationally lost COVID-19 cases. Journal of Microbiology, Immunology and Infection, 53(3), 454-58. https://www.sciencedirect.com/science/article/pii/S1684118220300736. DOI: https://doi.org/10.1016/j.jmii.2020.03.013.

Luan, X. et al. (2019) Exercise as a prescription for patients with various diseases. Journal of Sport and Health Science, 8(5), 422-41. https://www.sciencedirect.com/science/article/pii/S2095254619300493?via\%3Dihub. DOI: https://doi.org/10.1016/j.jshs.2019.04.002.

Lewnard, J. A. \& Lo, N. C. (2020) Scientific and ethical basis for social-distancing interventions against COVID-19. The Lancet Infectious Diseases, 20(6), 631-33. https://www.thelancet.com/journals/lancet/article/PIIS1473-3099(20)30190-0/fulltext. DOI: https://doi.org/10.1016/S1473-3099(20)30190-0.

Liz, C. M. \& Andrade, A. (2016). Análise qualitativa dos motivos de adesão e desistência da musculação em academias. Revista Brasileira de Ciências do Esporte, 38(3), 267-74. 
Research, Society and Development, v. 10, n. 13, e04101321045, 2021

(CC BY 4.0) | ISSN 2525-3409 | DOI: http://dx.doi.org/10.33448/rsd-v10i13.21045

MCkibbin, W. F. R. (2020). The Global Macroeconomic Impacts of COVID-19: Seven Scenarios. CAMA Working Paper, 19/2020. https://cama.crawford.anu.edu.au/publication/cama-working-paper-series/16221/global-macroeconomic-impacts-covid-19-seven-scenarios.

Neto, L. O. et al. (2020). \#TrainingInHome - Home-based training during COVID-19 (SARS-COV2) pandemic: physical exercise and behavior-based approach. Revista Brasileira de Fisiologia do Exercício, 19(2). https://www.portalatlanticaeditora.com.br/index.php/revistafisiologia/article/view/4006. DOI: https://doi.org/10.33233/rbfe.v19i2.4006.

Oliveira, C. E. P. (2011). Currículo do curso de Educação Física. UFV.

OECD. (2020). Global economy faces gravest threat since the crisis as coronavirus spreads. Organização para a Cooperação e o Desenvolvimento Econômico. https://www.oecd.org/newsroom/global-economy-faces-gravest-threat-since-the-crisis-as-coronavirus-spreads.htm?d=masukdist1.

Tojal, J. B. (1991). In Corpo movimento. APEF, II(III), 77.

Stein, E. (1999). A mulher: sua missão segundo a natureza e a graça. EDUSC, Bauru, SP.

Velavan, T. P. \& Meyer, C. G. (2020). The COVID-19 epidemic. Tropical Medicine \& International Health, 25(3), 278-80. https://pubmed.ncbi.nlm.nih.gov/ 32052514/. 10.1111/tmi.13383.

Wu, Y. et al. (2020). SARS-CoV-2 is an appropriate name for the new coronavirus. The Lancet, 395(10228), 949-50. https://www.thelancet.com/journa 1s/lancet/article/PIIS0140-6736(20)30557-2/fulltext. DOI: https://doi.org/10.1016/S0140-6736(20)30557-2. 